\title{
Too Much of a Good Thing: Weakness, Dysphagia, and Stridor After Botulinum Toxin Injections
}

Steven Dominguez, MD, MPH; Marek Dobke, MD, PhD; Steven Dominguez Jr, BS

\section{A 68-year-old woman presented with diminished ability to raise her head, difficulty swallowing, and intermittent stridor 5 days after receiving 225 IU of onabotulinumtoxinA.}

\section{Case}

A 68-year-old woman presented to the ED 5 days after receiving onabotulinumtoxinA cosmetic injections for wrinkles of the face and neck. She stated that she was unable to raise her head while in a supine position and that her head felt heavy when standing. She also experienced spasms and strain of the posterior cervical neck muscles. In addition, the patient described a constant need to swallow forcefully throughout the day, and felt an intermittent heavy sensation over her larynx that was associated with stridor. She noted these symptoms began 5 days after the onabotulinumtoxi$\mathrm{nA}$ injections and had peaked 2 days prior to presentation. She also complained of dysphagia without odynophagia, but denied any changes in her voice.

The patient first began onabotulinumtoxinA injections 12 years earlier for aesthetic treatment of glabellar and periorbital wrinkles. She initially received the injections at a regular interval of 90 to 100 days. During the course of the first 2 years of treatment, the patient was under the care of a plastic surgeon; thereafter, she sought treatment at a physician-owned medical spa because it offered onabotulinumtoxi$\mathrm{nA}$ at a lower price. The injections at the medical spa were administered by a physician assistant (PA). The patient stated that although the PA had steadily increased the dose of onabotulinumtoxinA to maintain the desired aesthetic effect, this was the first time she had experienced any side effects from the treatment.

The ED staff contacted the medical spa provider, who reviewed the patient's medical record over the telephone. The PA stated that he had been the only practitioner at the facility to administer the onabotulinumtoxinA injections to the patient over her past 10 years there as a client. He further informed the emergency physician (EP) that 12 days prior to presentation, he had given the patient a total of 50 IU of onabotulinumtoxinA, in five separate injections, into the mid frontalis muscle; a total of $35 \mathrm{IU}$, in seven separate injections,

Dr Dominguez is a plastic surgeon and retired emergency physician, Bella Milagros Institute, Downey, California. Dr Dobke is professor of surgery, and head, division of plastic surgery, University of California, San Diego. Mr Dominguez is a graduate student, Global Health Sciences, University of California, San Francisco.

Authors' Disclosure Statement: The authors report no actual or potential conflict of interest in relation to this article.

DOI: 10.12788/emed.2016.0076 


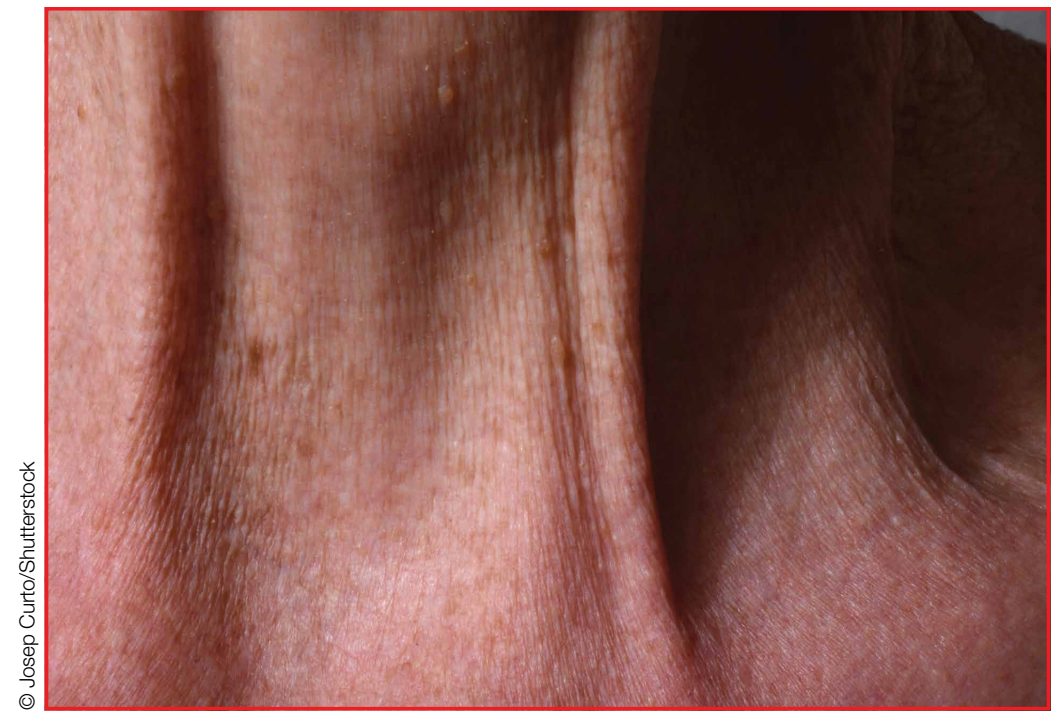

Figure. Photo (not of the patient discussed in this case report) shows anterior neck platysmal bands of varying length and width.

into the glabellar region (procerus and corrugator muscles bilaterally); 20 IU into the lateral and inferior-lateral orbicularis oculi bilaterally, in four separate injections per side, (40 IU total); and a total of $100 \mathrm{IU}$ in the anterior platysma, in 20 separate injections, for a total 1-day onabotulinumtoxinA dose of $225 \mathrm{IU}$.

The PA explained to the EP that he mixed the onabotulinumtoxinA in the patient's room and had shown her the vials and dilution standard as recommended by the manufacturer because she had been requiring increased dosages and had previously questioned whether the onabotulinumtoxinA was diluted. The PA denied any other patients experiencing similar adverse events as those of the patient's.

Over the last 10 years, the patient had received onabotulinumtoxinA in the nasolabial folds, upper and lower lip wrinkles, mentalis, depressor angular oris, buccal, nasalis, lateral brow, masseter, and calf muscles. The dosage of onabotulinumtoxinA at this most recent injection cycle was unchanged from her previous visit 3 months prior. According to the PA, the practice did not use abobotulinumtoxinA or incobotulinumtoxinA.
Regarding the patient's medical history, she had no health issues suggestive of myasthenia gravis, multiple sclerosis, or Guillain-Barré syndrome. Examination of the face revealed decreased muscle excursion of the frontalis muscle from midbrow to mid-brow, and stair-step wrinkle formation bilaterally. The procerus muscle was very weak, and the corrugator muscles were moderately diminished in strength. The lateral orbicularis oculi were very weak at each canthus. The extra-ocular muscles were intact. She had full mandibular excursion, and powerful movement of the tongue. The oropharynx and floor of the mouth were normal. She was noted to purposefully swallow and extend her neck every 90 to 120 seconds to "clear her throat," though she did not drool and was able to handle her secretions and swallow fluids without aspiration. Her voice was normal and she was able to recite the letters "KKKKK," "OOOOO," and "EEEEE" in rapid fashion without breathiness or stridor. The rest of her facial muscles were normal.

While examining the patient, the EP asked her to refrain from swallowing whenever she extended her neck. Upon complying with this request, her neck extension precipitated swallowing and, by not swallowing, she did not accumulate secretions. Once during the examination, the patient began swallowing and breathing rapidly with stridor. This less than 15-second episode was abated by full-neck extensions, which relieved the patient's sensation of heaviness over the larynx. Her breathing and voice were normal immediately after this episode.

Examination of the anterior neck revealed four platysmal bands (Figure). One band measured $10 \mathrm{~cm}$ in length and extended from the mandible inferiorly; two bands measured $2 \mathrm{~cm}$ lateral to the midline bilaterally; and the fourth band extended 4 $\mathrm{cm}$ in length from the mandible immediately lateral to the longer platysmal band. The platysma and dermis were flaccid and 
redundant at rest and with exertion. The sternocleidomastoid muscles were weak with exertion. The larynx moved cephalad with swallowing. The posterior cervical neck and trapezius muscles were of normal tone and strength. No spasms or fasciculations were noted during the examination period.

While supine, the patient strained to lift her head and complained of a suffocating sensation over the larynx. She had no rashes or edema, and the remainder of the physical examination, vital signs, and pulse oximetry were normal. Laboratory evaluation, which included a complete blood count and serum electrolytes, was also normal.

An otolaryngologist consultation for laryngoscopy was obtained. After reviewing the patient's case, the otolaryngologist concluded that given the patient's history, intermittent stridor, and an absence of signs or symptoms suggestive of an impending upper airway obstruction (UAO), laryngoscopy was not warranted.

A plastic surgery consultation was then obtained. The patient's examination was as noted above, and her vital signs and pulse oximetry remained normal throughout her ED stay. Although botulinum and botulinum antibody titers were ordered, the patient refused testing due to cost concerns. She was discharged home by plastic surgery services with a diagnosis of floppy neck and dysphagia secondary to aesthetic botulinum toxin paralysis of the bilateral sternocleidomastoid muscles and platysma. She was given a prescription for metoclopramide hydrochloride to stimulate motility of the upper gastrointestinal tract and to potentially improve swallowing. ${ }^{10}$

The patient was scheduled for a followup evaluation with the plastic surgeon 2 days after discharge. She was instructed to call 911 if she experienced stridor, shortness of breath, drooling, or if any airway issues arose. The patient did not return for her follow-up appointment with the plastic surgeon.
Table. Botulinum Toxins: Clinical Indications

\begin{tabular}{ll}
\hline Botulinum Type & Indication(s) \\
\hline $\begin{array}{l}\text { Botulinum toxin A } \\
\text { AbobotulinuntoxinA } \\
\text { IncobotulinumtoxinA } \\
\text { OnabotulinumtoxinA }\end{array}$ & $\begin{array}{l}\text { Aesthetic wrinkles, blepharospasm, } \\
\text { hyperhidrosis, migraine headache, } \\
\text { laryngeal dystonia, torticollis, and } \\
\text { overactive bladder }\end{array}$ \\
\hline Botulinum toxin B & \\
RimabotulinumtoxinB & Adults with cervical dystonia \\
\hline
\end{tabular}

\section{Discussion}

\section{Clostridium Botulinum Toxins}

Clostridium botulinum is a gram-positive spore-forming anaerobic bacterium that produces extremely potent neuro-exotoxins. $C$ botulinum is found in soil, contaminated foods, and in illicit injectable drugs (eg, heroin). Seven distinct antigenic botulinum toxins (A, B, C1, D, E, F, and $\mathrm{G}$ ) are produced by several strains of $C$ botulinum. Systemically, each neurotoxin is able to produce severe morbidity and mortality by causing generalized muscle paralysis and death by respiratory failure. The lethal dose of these agents is approximating 10(-9) g/kg body weight. Botulinum toxin type $\mathrm{A}$ is the most potent. ${ }^{1,2}$

Nonetheless, botulinum toxin has been used clinically since the early 1970s. Currently, there are three FDA-approved botulinum toxin type A agents and one type B formulation (rimabotulinumtoxinB) (Table). Each formulation is unique, proprietary, and differs in molecular weight, toxin-complex size, protein content, and inactive ingredients. The effectiveness and adverse event profile for these four botulinum toxins is individually dependent upon the different dilutions and potency, onset of action, duration of effect, diffusion, and migration potential. Hence, the effective dose of one botulinum toxin does not equate to any other, resulting in a lack of in- 
terchangeability between botulinum toxins (eg, 5 IU of incobotulinumtoxinA does not equal 5 IU of onabotulinumtoxinA).

\section{Aesthetic Indications}

Historically, the use of botulinum toxin for aesthetic treatment of wrinkles and platysmal bands was first reported by Blitzer ${ }^{3}$ in 1993. Subsequently, the use of botulinum toxin for the aesthetic treatment of facial wrinkles, hypertrophic platysmal bands and horizontal neck lines gained popularity within the public and medical community. ${ }^{3-5}$

Anatomically, the platysma is a thin

\section{The use of botulinum toxin for chemodenervation of the platysma can produce significant weakness of other neck muscles....}

sheet-like muscle that originates in the superior fascia of the pectoralis and deltoid fascia, and extends over the full length of the neck up past the mandible and continuing into the submuscular aponeurotic system. The platysma is innervated by the seventh cranial nerve and functions to pull the jaw downward. The platysma muscle is attached directly to the skin. With normal aging, the anterior neck skin becomes flaccid, the central platysmal bands thicken and contract-forming bands, horizontal wrinkles, and loss of definition of the neck noticed at rest and with contraction of the platysma muscle. These vertical bands are known as platysmal bands. The platysmal bands are benign consequences of aging and as such are targets of correction through surgery or botulinum toxin injection. ${ }^{6,7}$

\section{Mechanism of Action}

Platysmal band and horizontal line injection techniques with botulinum toxin have been reported in the literature with dosages ranging from 15 IU to 200 IU used to block the Soluble N-ethylmaleimide-sensitive factor activating protein receptors. Typical onset of action begins at 3 days, with full paralytic effect at 7 days. Repeat injections every 3 to 4 months are required with prolonged effects seen with each subsequent injection due to chemodenervation-induced muscle atrophy. ${ }^{4,7,8}$

\section{Adverse Effects}

Commercial botulinum toxin type A has been associated with minor and transient side effects. Moderate complications seen in the neck region include transient softtissue edema, dermal ecchymoses, intramuscular hematoma, diffuse muscle soreness, neck flexor weakness, and headaches. ${ }^{4,8,9}$

The use of botulinum toxin for chemodenervation of the platysma can produce significant weakness of other neck muscles, including the sternocleidomastoid, cricothyroid, sternothyroid, and sternohyoid. Floppy neck and dysphagia may be due to diffusion of the toxin into the muscles of deglutition of the larynx; injection directly into the sternocleidomastoid muscle; or a result of the systemic effects of large dosages. Hoarseness, breathiness, and dysphagia may occur 3 to 4 days after injection, especially with doses over 75 IU. $^{10}$

The recommended concentration of botulinum toxin type A causes a diffusion average of $1 \mathrm{~cm}$ in all directions from the injection sites. However, as the dilution increases, so does the zone of diffusion. Typical discharge instructions for platysma treatment include the overuse of the neck muscles for 2 to 4 hours after injection to encourage the botulinum toxin uptake for optimal result. Site manipulation (rubbing or massaging) also increases diffusion. For botulinum toxin type $\mathrm{B}$, the zone of diffusion is greater because its molecular weight is less than the type A toxins, thus making it an undesirable agent for aesthetic facial chemodenervation. ${ }^{4,11}$ 


\section{Toxin Resistance}

Botulinum toxin resistance is a known complication that occurs normally as a result of the body recognizing the neurotoxin as a foreign substance and producing neutralizing antibodies (NAb). Primary botulinum toxin failure is known in patients who require high doses of the neurotoxin for treatment of neuromuscular disorders. ${ }^{12}$ Complete secondary therapy failure is known to occur in cosmetic patients after a single dose and those who have been receiving low-dose botulinum toxin regularly. The risk of NAb development increases with long-term treatment and high doses. ${ }^{12-18}$

\section{Floppy Neck and Dysphagia}

As previously noted, floppy neck and dysphagia are adverse clinical findings of botulinum toxin effect on the platysma, sternocleidomastoid, or the paralaryngeal muscles. In this case, the patient was fortunate to have only sustained weakness of the platysma and sternocleidomastoid muscles despite both a large neck and total body dose. Paralaryngeal muscle paralysis is not life-threatening, but the distress may precipitate paradoxical vocal cord motion and stridor.

\section{Stridor}

Stridor is typically a symptom of an upper airway obstruction (UAO) process. Typical UAO conditions encountered in the ED are infections (eg, epiglottitis, croup), foreign body, allergy, and laryngeal trauma. The age of the patient, onset of stridor, course of the stridor (ie, intermittent, continuous, worsening), associated symptoms (eg, fever, rash, swelling of oral soft tissues), and bruising must be ascertained.

In differentiating the etiology of stridor, the EP should observe the patient for any associated change in voice, inability to handle secretions, and position of comfort. Patients with stridor require admission and evaluation by an otolaryngologist as expeditiously as possible because impending UAO may quickly progress to complete UAO necessitating emergent intubation.

An atypical presentation of stridor to the ED is sporadic stridor. Sporadic attacks of stridor during activity have been associated with the entity of paradoxical vocal cord motion. Patients usually describe a choking sensation with inability to breathe resulting in an audible inspiratory and/ or expiratory sound-ie, stridor. Wheezing may or may not be present. Patients may also describe tightness in the neck and sometimes in the chest. The attacks are usually seconds to minutes in duration. More often, there is a precipitating or an inducing factor such as hyperventilation, cough, panting, phonatory tasks, or the inhalation of irritants or perfume, or an oropharyngeal or laryngeal manipulation prior or postextubation. The feeling of stress alone is commonly reported prior to the attacks. When evaluating patients presenting with floppy neck, dysphagia, and stridor, it is imperative that the clinician conduct a thorough history and physical examination to determine if the symptoms are secondary to a systemic or local effect, and whether the patient will progress to an acute UAO (vocal cord paralysis) necessitating intubation in the ED and subsequent tracheostomy. ${ }^{19,20}$

\section{Conclusion}

The ready availability of botulinum toxins and their low-cost-benefit ratio continue to promote over-utilization for treatment of facial wrinkles, platysmal bands, and horizontal lines; migraine headache; and hyperhidrosis. Complications associated with overuse of botulinum toxins are due to either administration of a large single dose or from regional diffusion. With the increasing number of patients receiving botulinum injections, EPs should be aware of the four available toxin types onset of action, adverse events, and potential lifethreatening complications of regional neck injections. 


\section{References}

1. Huang W, Foster JA, Rogachefsky AS. Pharmacology of botulinum toxin. J Am Acad Dermatol. 2000;43(2 Pt 1):249-259. doi:10.1067/mjd.2000.105567.

2. Lamanna C. The most poisonous poison. Science. 1959;130(3378):763-772.

3. Blitzer A, Brin MF, Keen MS, Aviv JE. Botulinum toxin for the treatment of hyperfunctional lines of the face. Arch Otolaryngol Head Neck Surg. 1993;119(9):1018-1022.

4. Carruthers A, Carruthers J. Clinical indications and injection technique for the cosmetic use of botulinum A exotoxin. Dermatol Surg. 1998;24(11):1189-1194.

5. Carruthers J, Carruthers A. Botox use in the mid and lower face and neck. Semin Cutan Med Surg. 2001;20(2):85-92. doi:10.1053/sder.2001.25139

6. Hoefflin SM. Anatomy of the platysma and lip depressor muscles. A simplified mnemonic approach. Dermatol Surg. 1998;24(11):1225-1231.

7. Brandt FS, Bellman B. Cosmetic use of botulinum A exotoxin for the aging neck. Dermatol Surg. 1998;24(11):1232-1234

8. Klein AW. Complications and adverse reactions with the use of botulinum toxin. Semin Cutan Med Surg. 2001;20(2):109-120. doi:10.1053/sder.2001.25964.

9. Carruthers A, Kiene K, Carruthers J. Botulinum A exotoxin use in clinical dermatology. J Am Acad Dermatol. 1996;34(5 Pt 1):788-797.

10. Howell K, Selber P, Graham HK, Reddihough D. Botulinum neurotoxin A: an unusual systemic effect. J Paediatr Child Health. 2007:43(6):499-501. doi:10.1111/j.1440-1754.2007.01122.x.

11. Carruthers A, Carruthers J. Toxins 99, new information about the botulinum neurotoxins. Dermatol Surg. 2000;26(3):174-176.
12. Dressler D, Adib Saberi F. New formulation of Botox: complete antibody-induced treatment failure in cervical dystonia. J Neurol Neurosurg Psychiatry. 2007;78(1):108-109. doi:10.1136/jnnp.2006.093419.

13. Borodic G. Immunologic resistance after repeated botulinum toxin type a injections for facial rhytides. Ophthal Plast Reconstr Surg. 2006;22:239-240. doi:10.1097/01.iop.0000217703.80859.a3.

14. Goschel H, Wohlfarth K, Frevert J, Dengler R, Bigalke $\mathrm{H}$. Botulinum A toxin therapy: neutralizing and nonneutralizing antibodies-therapeutic consequences. Exp Neurol. 1997;147(1):96-102. doi:10.1006/ exnr.1997.6580.

15. Hatheway CL. Toxigenic clostridia. Clin Microbiol Rev. 1990;3(1):66-98.

16. Smith LA. Development of recombinant vaccines for botulinum neurotoxin. Toxicon. 1998;36(11):1539-1548.

17. Houser MK, Sheean GL, Lees AJ. Further studies using higher doses of botulinum toxin type $\mathrm{F}$ for torticollis resistant to botulinum toxin type A. J Neurol Neurosurg Psychiatry. 1998;64(5):577-580.

18. Dressler D, Wohlfahrt K, Meyer-Rogge E, Wiest L, Bigalke H. Antibody-induced failure of botulinum toxin a therapy in cosmetic indications. Dermatol Surg. 2010;36 Suppl 4:2182-2187. doi:10.1111/ j.1524-4725.2010.01710.x.

19. Maschka DA, Bauman NM, McCray PB Jr, Hoffman HT, Karnell MP, Smith RJ. A classification scheme for paradoxical vocal cord motion. Laryngoscope. 1997;107(11 Pt 1):1429-1435.

20. Altman KW, Simpson CB, Amin MR, Abaza M, Balkissoon R, Casiano RR. Cough and paradoxical vocal fold motion. Otolaryngol Head Neck Surg. 2002;127(6):501-511. doi:10.1067/mhn.2002.127589. 